\title{
Supporting Information: Integrating Machine Learning with Multi-Layer Energy-Based Fragment Method for Excited States of Large Systems
}

\author{
Wen-Kai Chen, Wei-Hai Fang, and Ganglong Cui*
}

Key Laboratory of Theoretical and Computational Photochemistry, Ministry of Education, College of Chemistry, Beijing Normal University, Beijing 100875, China

Email: ganglong.cui@bnu.edu.cn

\section{Contents}

1 Ab Initio Methods $\quad S 2$

2 ML Data Preparation $\quad$ S2

3 Local Frame Descriptor $\quad$ S3

4 Deep Neural Networks $\quad$ S4

5 Training Parameters $\quad$ S5

6 Additional ML Results $\quad$ S6

7 Additional ML-MLEBF Results $\quad$ S7

8 Zhu-Nakamura Method $\quad$ S8

9 Additional Nonadiabatic Dynamics Results $\quad$ S9

10 Simplified ML-MLEBF Method $\quad S 12$

11 Comparison between MLEBF and QM/MM method S14 


\section{Ab Initio Methods}

In this work we have used 200 structures of $\mathrm{CH}_{3} \mathrm{~N}=\mathrm{NCH}_{3}-\left(\mathrm{H}_{2} \mathrm{O}\right)_{5}$ as benchmark data for testing the accuracy of ML-MLEBF calculated energies and gradients. These structures are randomly selected from a 2 ps canonical molecular dynamics trajectory performed at the BP86/def2-SVP level. ${ }^{1-3}$ Time step of $1 \mathrm{fs}$ is used for nuclear propagation and the five-order Nosé-Hoover chain thermostat technique ${ }^{4,5}$ is used to control the temperature in canonical molecular dynamics simulations $(\mathrm{T}=300 \mathrm{~K})$, which is performed using Chemshell3.5 $5^{6-8}$ interfaced with Turbomole6.6. ${ }^{9-11}$

In our ML-MLEBF calculations, the inner region i.e. photochemically active region is treated with CASSCF and the inter-layer interaction (dimers and trimers) between both inner and outer regions is also done at the same computational level to consider their mutual polarization. In state-averaged CASSCF/6-31+G** calculations, two roots with same weights are used, together with an active space of six electrons in four orbitals, which contains pi and pi ${ }^{*}$ orbitals of $\mathrm{N}=\mathrm{N}$ and two lone-pair orbitals on the two nitrogen atoms of $\mathrm{CH}_{3} \mathrm{~N}=\mathrm{NCH}_{3} .{ }^{12}$ This selection provides good results. ${ }^{13}$ All SA-CASSCF calculations are done with MOLCAS8.0. ${ }^{14,15}$

\section{ML Data Preparation}

In contrast to previous studies, where the efficient DFT method is used, ${ }^{13}$ in the present work, the outer surrounding region i.e. photochemically inert region is described with the more efficient $\mathrm{ML}$ models that will be trained by the deep neural network (DNN) method. ${ }^{16-33}$ To train the ML models for monomer energy $E_{i}$, and dimer and trimer interaction energies $\triangle E_{i j}$ and $\triangle E_{i j k}$ of water molecules, we have prepared the training, testing, and validation structures through molecular dynamics simulations. We have performed a 500 ps canonical molecular dynamics of 112 waters in a cubic box of $17^{*} 17^{\star} 17 \AA$ with 0.5 fs time step at $300 \mathrm{~K}$. In the simulation, the simple SPC water model is used for water molecules and there are no any internally rigid constraints on waters. ${ }^{34,35}$ We record one snapshot every 500 steps and totally obtain 2000 snapshots, from which the training, testing, and validation structures of monomers, dimers, and trimers are constructed (see Table S1). Then, energies and gradients of these structures are calculated at the B3LYP/6-31+G** level $^{2,36-41}$ and used as reference data for machine learning (see above). Ambertools 17 is used for MD simulations ${ }^{42}$ while Gaussian16 is done for B3LYP single point calculations. ${ }^{43}$

Table S1: Numbers of Data Sets of Monomers, Dimers, and Trimers used in the Deep Learning.

\begin{tabular}{|c|c|c|c|}
\hline & monomer & dimer & trimer \\
\hline \hline training set & 144,000 & 100,000 & 60,000 \\
\hline testing set & 36,000 & 10,000 & 6,000 \\
\hline validation set & 36,000 & 10,000 & 6,000 \\
\hline
\end{tabular}




\section{Local Frame Descriptor}

For neural networks it is critical to get a good molecular descriptor that can preserve rotational, transitional, and permutational symmetries of a molecular system. As done in our previous work, we have used the local frame molecular descriptor developed by Weinan E et al. ${ }^{25}$ Here a brief presentation is given to form a self-contained work. Details are referred to the original literature. ${ }^{25}$

In this molecular descriptor, a cutoff radius $R_{c}$ is introduced as the only parameter, which make its accuracy capable of systematically improved by increasing the threshold value. The molecular structure under a laboratory frame $\left(\left\{\mathbf{R}_{i}^{l a b}\right\}=\left\{x_{i}^{l a b}, y_{i}^{l a b}, z_{i}^{l a b}\right\}\right)$ i.e. Cartesian coordinate system will be rotated to a local frame $\left(\left\{\mathbf{R}_{i}\right\}=\left\{x_{i}, y_{i}, z_{i}\right\}\right)$. Next we use labels with a superscript lab for physical quantities under the laboratory frame and labels without any superscript for those under the local frame. A set of neighbor atoms for the atom $i$ is labeled as $\{N(i)\}=\left\{j:\left|R_{i j}^{l a b}\right|<R_{c}\right\}$, where $R_{i j}^{l a b}=\left|\mathbf{R}_{i j}^{l a b}\right|=\left|\mathbf{R}_{i}^{l a b}-\mathbf{R}_{j}^{l a b}\right|$. Two atoms called axis-atoms and expressed by $a(i) \in\{N(i)\}$ and $b(i) \in\{N(i)\}$ are selected by users to define the axis of a local frame of each atom $i$. The closest atoms of two different elements are usually chosen as the axis-atoms. These two atoms $a(i), b(i)$ together with the center atom $i$ define a local frame $\left\{\mathbf{e}_{i 1}, \mathbf{e}_{i 2}, \mathbf{e}_{i 3}\right\}$ as following:

$$
\begin{gathered}
\mathbf{e}_{i 1}=\mathbf{e}\left(\mathbf{R}_{i a(i)}^{l a b}\right), \\
\mathbf{e}_{i 2}=\mathbf{e}\left(\mathbf{R}_{i b(i)}^{l a b}-\left(\mathbf{R}_{i b(i)}^{l a b} \cdot \mathbf{e}_{i 1}\right) \mathbf{e}_{i 1}\right), \\
\mathbf{e}_{i 3}=\mathbf{e}_{i 1} \times \mathbf{e}_{i 2},
\end{gathered}
$$

where $e(\mathbf{R})$ is the function that normalizes the vector $\mathbf{R}$, i.e. $\mathbf{e}(\mathbf{R})=\mathbf{R} /|\mathbf{R}|$. With the local frame $\left\{\mathbf{e}_{i 1}, \mathbf{e}_{i 2}, \mathbf{e}_{i 3}\right\}$ we could determine the rotation matrix $\mathscr{R}\left(\mathbf{R}_{i a(i)}^{l a b}, \mathbf{R}_{i b(i)}^{l a b}\right)$ by the following equation:

$$
\mathscr{R}\left(\mathbf{R}_{i a(i)}^{l a b}, \mathbf{R}_{i b(i)}^{l a b}\right)=\left(\begin{array}{c}
\mathbf{e}_{i 1} \\
\mathbf{e}_{i 2} \\
\mathbf{e}_{i 3}
\end{array}\right)^{T}
$$

After that we could get the coordinates under the local frame

$$
\mathbf{R}_{i j}=\mathbf{R}_{i j}^{l a b} \mathscr{R}\left(\mathbf{R}_{i a(i)}^{l a b}, \mathbf{R}_{i b(i)}^{l a b}\right)
$$


in which $R_{i j}=\left|\mathbf{R}_{i j}\right|$. Finally, the molecular descriptor $\left\{\mathbf{D}_{i j}\right\}$ is expressed as

$$
\left\{\mathbf{D}_{i j}\right\}= \begin{cases}\left\{\frac{1}{R_{i j}}, \frac{x_{i j}}{\left(R_{i j}\right)^{2}}, \frac{y_{i j}}{\left(R_{i j}\right)^{2}}, \frac{z_{i j}}{\left(R_{i j}\right)^{2}}\right\}, & \text { full-information } ; \\ \left\{\frac{1}{R_{i j}}\right\}, & \text { radial-only-information } .\end{cases}
$$

In our work, we use a local frame descriptor with full information as input of DNNs. Through preliminary testing, we have chosen a cutoff radius of $R_{c}=12 \AA$, which is demonstrated to be good enough for our ML models. This molecular descriptor has been recently used to train multi-state potential energy surfaces with conical intersections and is shown to be able to give correct description for excited states and photodynamics. ${ }^{28}$

\section{Deep Neural Networks}

In this work, we have built three ML models for water molecules, which correspond to their monomer energy $E_{i}$, and dimer and trimer interaction energies $\triangle E_{i j}$ and $\triangle E_{i j k}$ (see main text for their definitions). For each $\mathrm{ML}$ model, we have used a set of feed-forward DNNs to learn the corresponding potential energy surfaces. To be convenient, we have used a simple $E$ to represent $E_{i}, \triangle E_{i j}$, and $\triangle E_{i j k}$ in the following expressions.

The potential energy of a single atomic configuration $E$ is expressed as the sum of $N_{\text {atom }}$ atomic energy contributions $E_{i}{ }^{16,22}$

$$
E=\sum_{i}^{N_{\text {atom }}} E_{i}
$$

in which each atomic energy $E_{i}$ is obtained by two steps. First, the descriptor $\left\{\mathbf{D}_{i j}\right\}$ of the $i$ th atom is constructed according to its coordinates under the lab frame as well as its neighbor atoms in the neighbor list $N(i)$. Once we get the descriptor $\left\{\mathbf{D}_{i j}\right\}$, we could use it as input of the element-specific DNN, which will give the prediction of the atomic energy,

$$
E_{i}=\mathbb{F}_{i}^{\text {out }}\left(\mathbb{F}_{i}^{h}\left(\mathbb{F}_{i}^{h-1}\left(\cdots\left(\mathbb{F}_{i}^{1}\left(\left\{\mathbf{d}_{i}^{0}\right\}\right)\right)\right)\right)\right)
$$

in which $\mathbb{F}_{i}^{h}\left(\mathbf{d}_{i}^{h-1}\right)=\psi\left(\mathbf{W}_{i}^{d} \mathbf{d}_{i}^{h-1}+\mathbf{b}_{i}^{h}\right)$ is a combination of a linear transformation and non-linear transformation where $\mathbf{d}_{i}^{h} \in \mathbb{R}^{n_{h}}$ is the vector denoting the values of neurons in the layer $h$ and $n_{h}$ is the number of related nodes. The weight matrix $\mathbf{W}_{i}^{d} \in \mathbb{R}^{n_{h} \times n_{h-1}}$ and bias vector $\mathbf{b}_{i}^{h} \in \mathbb{R}^{n_{h}}$ are parameters to be optimized. The nonlinear function $\psi$ known as activation function is taken to be hyperbolic tangent functions i.e. 


$$
\psi\left(d_{1}, d_{2}, \ldots, d_{n}\right)=\left(\tanh \left(d_{1}\right), \tanh \left(d_{2}\right), \ldots, \tanh \left(d_{n}\right)\right) .
$$

However, the output layer $\mathbb{F}_{i}^{\text {out }}\left(\mathbf{d}_{i}^{h}\right)=\mathbf{W}_{i}^{\text {out }} \mathbf{d}_{i}^{h}+\mathbf{b}_{i}^{\text {out }}$ is merely a linear transformation without activation functions, which is is used to avoid any restriction in the range of possible output values of DNNs. Overall, all parameters related to the layer $i$ can be expressed as

$$
\omega(i)=\left\{\mathbf{W}_{i}^{1}, \mathbf{b}_{i}^{1}, \mathbf{W}_{i}^{2}, \mathbf{b}_{i}^{2}, \ldots, \mathbf{W}_{i}^{\text {out }}, \mathbf{b}_{i}^{\text {out }}\right\}
$$

Finally, same elements share same parameters, which preserves the permutational symmetry. Numerical values of these parameters in DNNs determine the capability of DNNs to represent PESs. These parameters are optimized iteratively to improve the performance of DNNs. This process corresponds to minimizing the loss function. In this work, the loss function is defined as following:

$$
\mathbb{L}\left(p_{e}, p_{f}\right)=p_{e} \Delta E^{2}+\frac{p_{f}}{3 N_{\text {atom }}} \sum_{i}\left|\Delta F_{i}\right|^{2}
$$

In this function, $p_{e}$ and $p_{f}$ are prefactors used to speed up the training process and are defined as

$$
p=p_{\text {limit }}\left(1-\frac{l r}{l r_{0}}\right)+p_{\text {start }}\left(\frac{l r}{l r_{0}}\right)
$$

in which $p_{\text {start }}$ is the prefactor used at the beginning of the training process, and $p_{\text {limit }}$ is the approximate prefactor at the end. $l r_{0}$ means the initial learning rate and $l r$ is the current learning rate during the training process. The current learning rate $l r$ decays exponentially with the global step:

$$
l r=l r_{0} d_{r}^{-c_{s} / d_{s}}
$$

in which $d_{r}, c_{s}$ and $d_{s}$ are the decay rate, the global step, and the decay step, respectively. More details can be found in the original literature ${ }^{25}$ and our previous work. ${ }^{28}$

\section{Training Parameters}

In the training we use the energy prefactors $p_{\text {start }}=0.02$ and $p_{\text {limite }_{e}}=8$ and the force prefactors $p_{\text {start }}=1000$ and $p_{\text {limit }}=1$. These settings are based on the following consideration. Each molecule has one energy data 
and $3 * N_{\text {atom }}$ force data, which leads to a larger number of forces than energies. Thus, minimizing the loss of forces at the beginning of the training process could improve the training efficiency. Increasing the prefactors of energy in the training process could give a good balance between energy and force. The algorithm of Adam stochastic gradient descent method is used via external tensorflow package. The initial learning rate $l r_{0}$ is set to 0.0001 while the decay rate is set to 0.96 , which is updated every 500 steps in the training process of 100,000 steps. In order to avoid falling into local minima, the mini-batch training trick is used. In our work we have used a deep neural network with 8 hidden layers with 1600, 1600, 800,600, 400, 200, 100, 30 nodes. The training processes of the ML models are performed using the deepmd-kit program developed by $\mathrm{E}$ et al. ${ }^{44}$ Once the ML models are trained, we have used independent data for validating the accuracy of our trained ML models. Finally, it should be noted that we have used same training parameters for the three ML models for monomer energy $E_{i}$, and dimer and trimer interaction energies $\triangle E_{i j}$ and $\triangle E_{i j k}$.

Table S2: RMSDs of Energies and Forces of Monomers, Dimers, and Trimers for Training, Testing, and Validation Sets

\begin{tabular}{|c|c|c|c|}
\hline \multicolumn{4}{|c|}{ Energy RMSD $(\mathrm{eV})$} \\
\hline & Training Set & Testing Set & Validation Set \\
\hline Monomer & $1.06 \times 10^{-4}$ & $7.78 \times 10^{-5}$ & $7.92 \times 10^{-5}$ \\
\hline Dimer & $2.73 \times 10^{-4}$ & $2.46 \times 10^{-4}$ & $2.99 \times 10^{-4}$ \\
\hline Trimer & $2.75 \times 10^{-4}$ & $3.99 \times 10^{-4}$ & $4.58 \times 10^{-4}$ \\
\hline \multicolumn{4}{|c|}{ Forces RMSD $(\mathrm{eV} / \AA)$} \\
\hline & Training Set & Testing Set & Validation Set \\
\hline Monomer & $1.06 \times 10^{-3}$ & $9.45 \times 10^{-4}$ & $1.03 \times 10^{-3}$ \\
\hline Dimer & $1.03 \times 10^{-3}$ & $1.00 \times 10^{-3}$ & $1.23 \times 10^{-3}$ \\
\hline Trimer & $4.01 \times 10^{-4}$ & $8.71 \times 10^{-4}$ & $8.53 \times 10^{-4}$ \\
\hline
\end{tabular}

\section{Additional ML Results}

The trained ML models are very accurate and well reproduce the reference data. Table S2 collects RMSD values of energies and gradients of monomers, dimers, and trimers for training, testing, and validation sets. Fig. S1 shows comparison of energies and forces of all monomers, dimers, and trimers of training, testing, and validation sets between $M L$ models and ab initio reference data. 

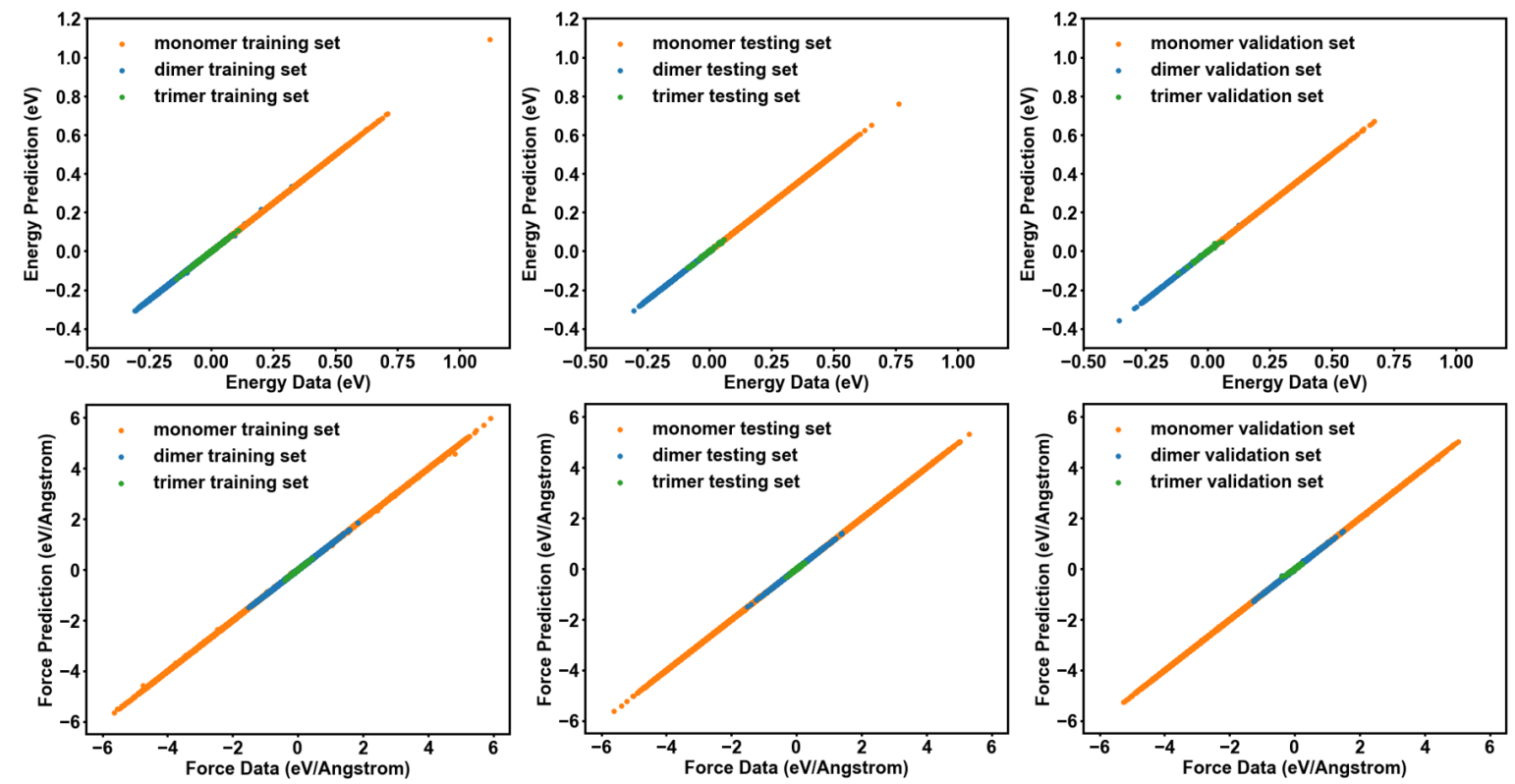

Figure S1: Comparison of energies and forces of monomers, dimers, and trimers of training, testing, and validation sets between ML models and ab initio reference data.

\section{Additional ML-MLEBF Results}

The ML-MLEBF method truncated at three-body interaction without electrostatically embedding gives accurate results (see discussion in the main text). RMSDs of $S_{0}$ and $S_{1}$ energies [gradients] are $4.83 \times 10^{-3} \mathrm{eV} /$ fragment

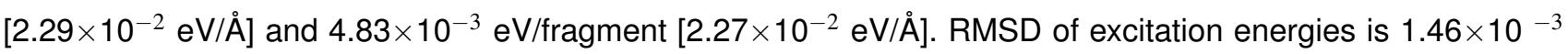
eV (see Fig. S2). 

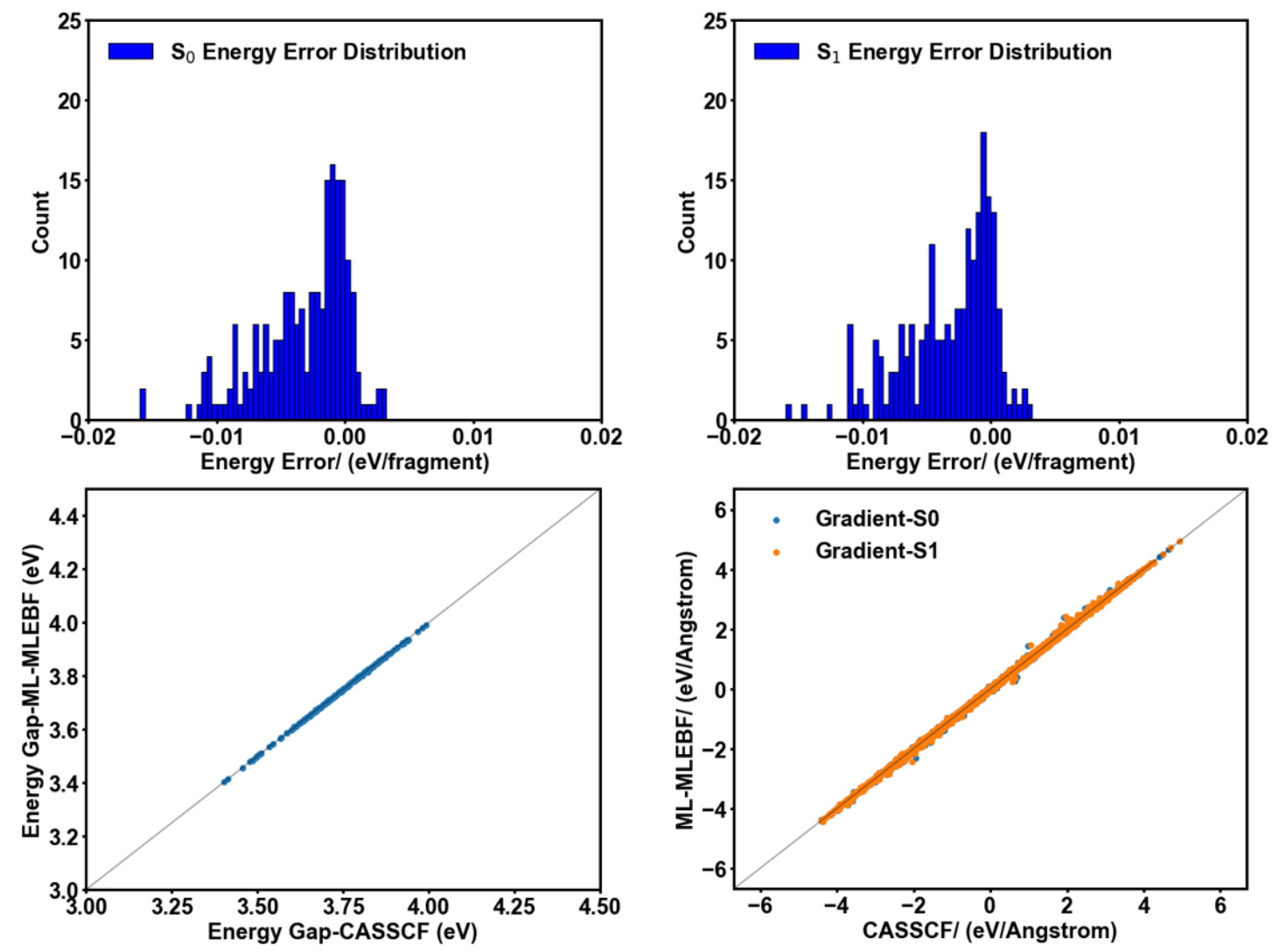

Figure S2: Comparison of both $\mathrm{S}_{0}$ and $\mathrm{S}_{1}$ energies (top-left and -right), energy gaps (bottom-left), and both $\mathrm{S}_{0}$ and $S_{1}$ gradients (bottom-right) calculated using ML-MLEBF and CASSCF.

\section{Zhu-Nakamura Method}

Trajectory-based surface hopping methods are one of popular methods that perform nonadiabatic dynamics simulations. In dynamics simulations, the system is propagated in an electronic state but can jump to other potential energy surfaces in particular near quasi-degenerate regions. In this work, we have used Zhu-Nakamura method to compute transition probabilities among different potential energy surfaces. This method is developed by Zhu et al. ${ }^{45-47}$ and is able to calculate nonadiabatic transition probabilities,

$$
p=\exp \left[-\left(\frac{\pi}{4 \sqrt{a^{2}}} \sqrt{\frac{2}{b^{2}+\sqrt{\left|b^{4} \pm 1\right|}}}\right)\right]
$$

in which $a$ and $b$ are two unitless parameters, i.e., effective coupling and collision energy, which are written as

$$
a^{2}=\frac{h^{2}}{8 \pi^{2} \mu} \frac{\sqrt{\left|F_{1} F_{2}\right|}\left|F_{2}-F_{1}\right|}{\left(2 V_{12}\right)^{3}}
$$


and

$$
b^{2}=\left(E_{t}-E_{x}\right) \frac{\left|F_{2}-F_{1}\right|}{\sqrt{\left|F_{2} F_{1}\right|}\left(2 V_{12}\right)},
$$

where $F_{1}$ and $F_{2}$ are two mass-scaled one-dimensional diabatic forces, $\mu$ is reduced mass of the diabatic molecule, $V_{12}$ is diabatic coupling, $E_{x}$ is the energy at crossing point, $E_{t}$ is potential energy plus kinetic energy component along hopping vector direction. Finally, mass-scaled one-dimensional diabatic forces in Eqs. S15 and S16 are converted from mass-scaled multidimensional diabatic forces based on

$$
\frac{\sqrt{\left|F_{2} F_{1}\right|}}{\sqrt{\mu}}=\sqrt{\left|\sum_{i=1}^{N} \frac{1}{m_{i}} \sum_{\alpha=x, y, z} F_{2}^{i \alpha} F_{1}^{i \alpha}\right|}
$$

and

$$
\frac{\left|F_{2}-F_{1}\right|}{\sqrt{\mu}}=\sqrt{\sum_{i=1}^{N} \frac{1}{m_{i}} \sum_{\alpha=x, y, z}\left(F_{2}^{i \alpha}-F_{1}^{i \alpha}\right)^{2}}
$$

in which $N$ is the number of atoms in a system; $m_{i}$ is the mass of the $i$ th atom; $F_{1}^{i \alpha}$ and $F_{2}^{i \alpha}$ are multi-dimensional diabatic forces of the $i$ th atom $(\alpha:, x, y$, and $z$ ) related to involved two states. These diabatic forces can be further converted from multi-dimensional adiabatic forces, which are directly calculated by external electronic structure packages. The detailed description can be found in recent works by Zhu and co-workers. ${ }^{48,49}$ The advantage of Zhu-Nakamura method compared with other surface-hopping methods is that it does not need to compute nonadiabatic coupling vectors.

Initial conditions of non-adiabatic molecular dynamics simulations are sampled via Wigner sampling, which is

performed by Newton-X. ${ }^{50}$ ? -52 In order to make comparison between CASSCF with ML-MLEBF method, same initial conditions are used in both kinds of dynamics simulations. For each trajectory, 500 fs propagation time with 0.5 fs time step are carried out using our GTSH package. ${ }^{53}$

\section{Additional Nonadiabatic Dynamics Results}

Fig. S3 shows time-dependent $S_{0}$ and $S_{1}$ state populations averaged over 100 trajectories, we could find that the state population is highly similar between CASSCF- and ML-MLEBF-based dynamics simulations. Fitting the $S_{1}$ state population with a single-exponential function of $y=\exp \left(-\left(t-t_{1}\right) / t_{2}\right)$ leads to $t_{1}=89$ and 89 and $t_{2}=128$ and $t_{2}=128 \mathrm{fs}$ for both CASSCF and ML-MLEBF dynamics simulations, respectively. 


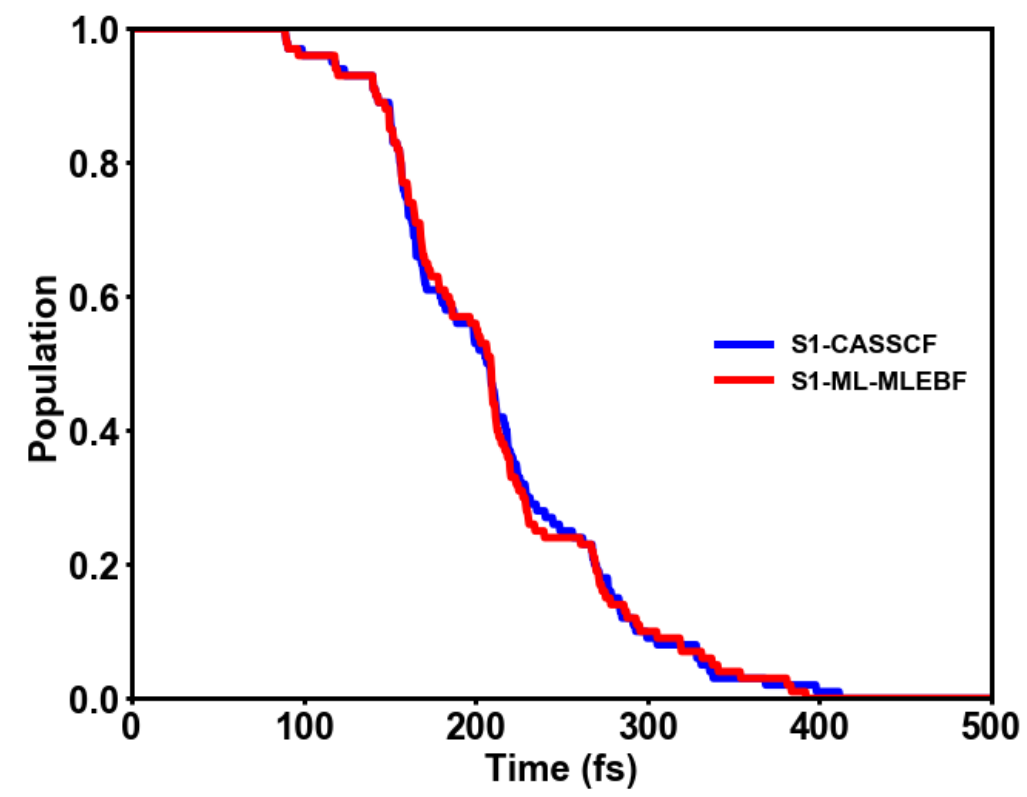

Figure S3: Time-dependent $S_{1}$ state population in full CASSCF- and ML-MLEBF-based dynamics simulations.

Dynamical results of both CASSCF and ML-MLEBF nonadiabatic dynamics simulations are pretty similar to each other. In addition to those discussed in the main text, the following results are also presented. Fig. S4 shows the distribution of the $S_{0}-S_{1}$ energy gap of 100 trajectories in the Franck Condon region, i.e. vertical excitation energies. ML-MLEBF well reproduces the CASSCF results. The same distribution width and the same peak are observed.

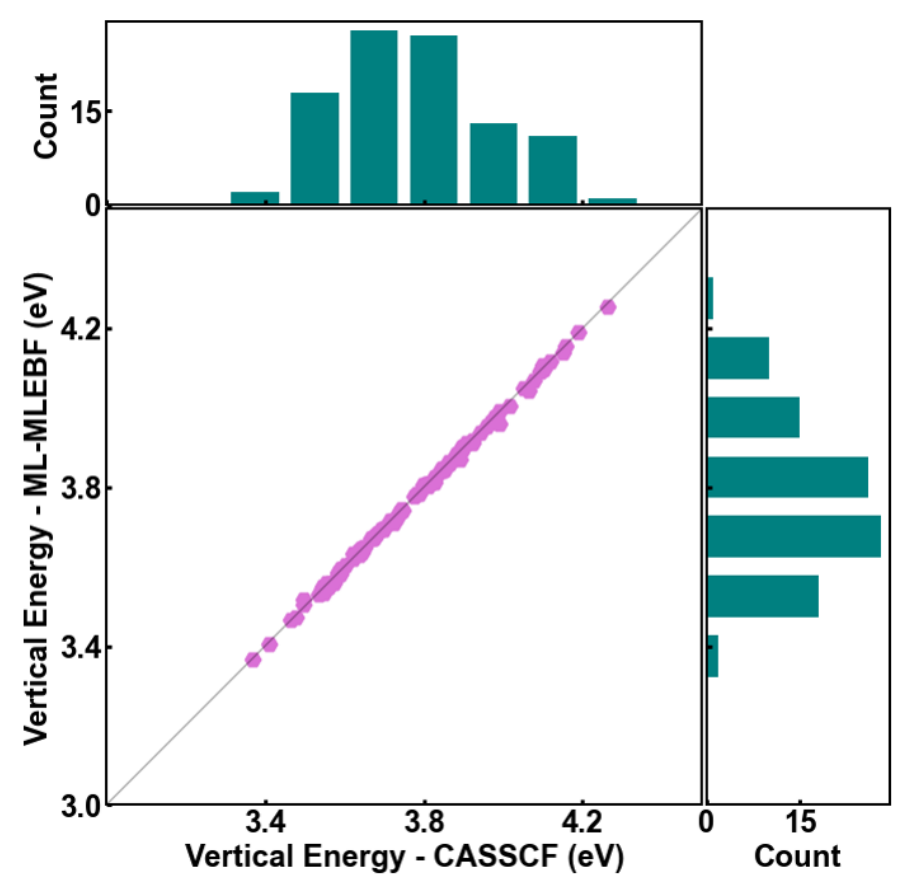

Figure S4: Comparison of the $\mathrm{S}_{0}-\mathrm{S}_{1}$ energy gap (eV) of 100 trajectories at the starting points calculated with full CASSCF and ML-MLEBF methods. 
Topological structures of potential energy surfaces (PES) of quasi-degenerate regions, e.g. conical intersections, play a crucial role in photochemistry and excited-state relaxation dynamics. Due to this reason, we have checked the reliability of ML-MLEBF in the description of the $\mathrm{S}_{1} / \mathrm{S}_{0}$ conical intersection of $\mathrm{CH}_{3} \mathrm{~N}=\mathrm{NCH}_{3}$. Fig S5 shows ML-MLEBF and CASSCF calculated two- and three-dimensional $\mathrm{S}_{0}$ and $\mathrm{S}_{1}$ potential energy surfaces expanded with regard to adiabatic derivative coupling $(\mathrm{h})$ and gradient difference $(\mathrm{g})$ vectors. Both methods give essentially same results (grid vs. surface in the left panel of Fig. S5). Additionally, in ML-MLEBF, the total energy is also conserved very well in comparison with CASSCF (see Fig. S6).
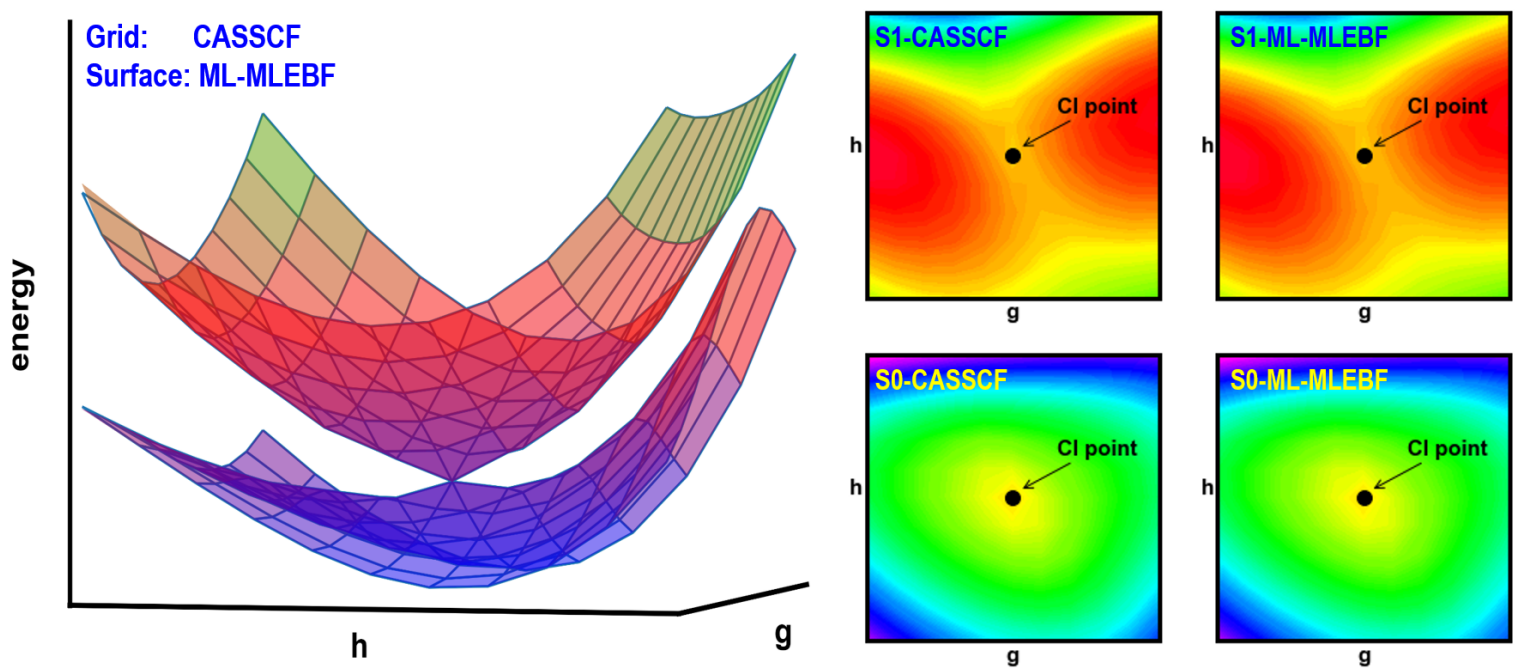

Figure S5: Two- and three-dimensional potential energy surfaces with respect to the two branching space vectors $g$ and $h$ near the $S_{1} / S_{0}$ conical intersection calculated with both ML-MLEBF and CASSCF methods.
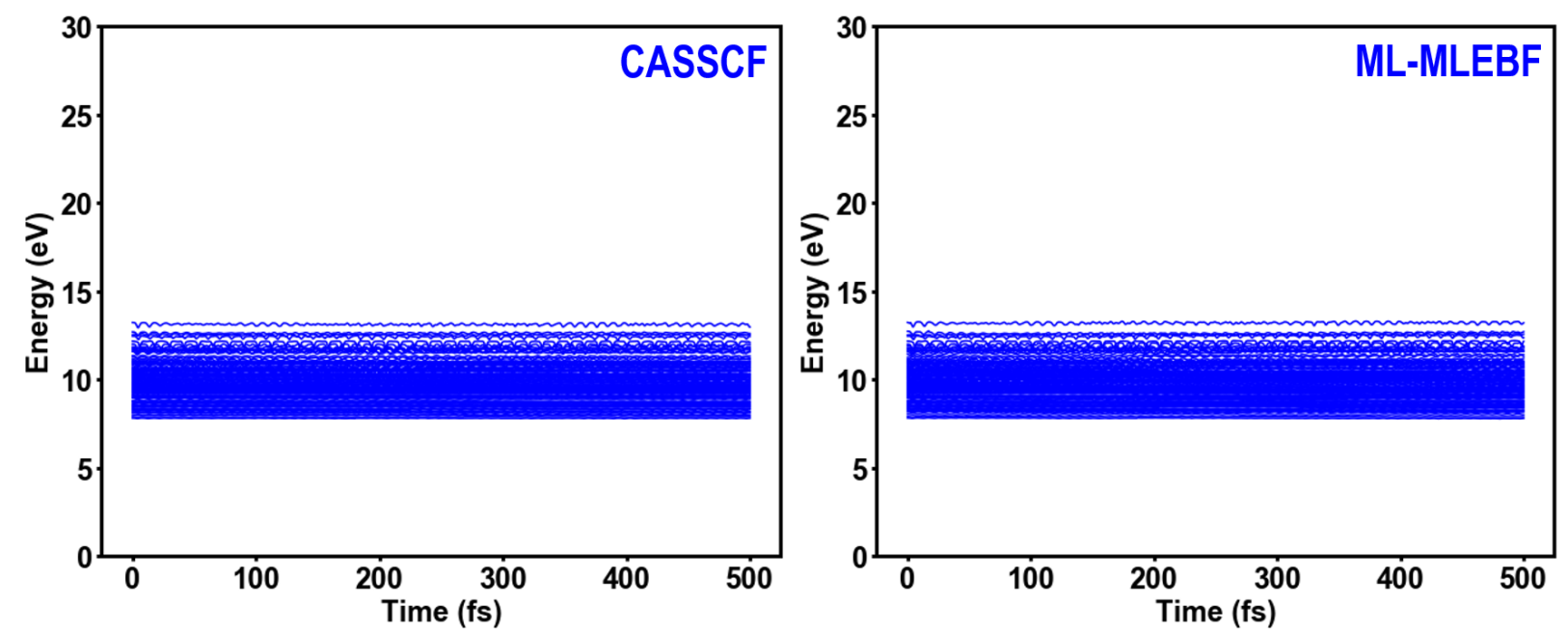

Figure S6: Time-dependent evolution of total energy of all trajectories in (left) CASSCF- and (right) MLEBFbased MD simulations. 


\section{Simplified ML-MLEBF Method}

In the ML-MLEBF method, discussed in the main text, the outer region is treated with the cheap but accurate ML models and thus it becomes much more efficient than full ab initio methods for large systems. However, the computational cost of the ML-MLEBF method can be further reduced by only including the two-body interaction for the intra-layer energy of the inner region and the inter-layer interaction between the inner and outer regions. In the simplified ML-MLEBF method, the total energy of a target system consisting of $N$ monomers is expressed

$$
E=E(\text { in })+E(\text { in } / \text { out })+E(\text { out })
$$

in which $E$ (in) and $E$ (out) are intra-layer energies of the inner and outer regions; $E$ (in/out) is the inter-layer interaction between the inner and outer regions; $N_{i n}$ represents the number of monomers in the inner region; $N_{\text {out }}$ represents the number of monomers in the outer region; $N$ represents the number of total monomers. In this scheme, the intra-layer interaction within the inner region, i.e. $E($ in $)$, and the inter-layer interaction between the inner and outer regions, i.e. E(in/out), are truncated at the two-body interaction level and treated with CASSCF in an electrostatically embedding field of fixed point charges, expressed as below:

$$
\begin{gathered}
E(\text { in })=\sum_{i<j}^{N_{\text {in }}} E_{i j}^{\prime}-\left(N_{\text {in }}-2\right) \sum_{i}^{N_{\text {in }}} E_{i}^{\prime} \\
E(\text { in } / \text { out })=\sum_{i}^{N_{\text {in }}} \sum_{j}^{N_{\text {out }}} \Delta E_{i j}^{\prime}=\sum_{i}^{N_{\text {in }}} \sum_{j}^{N_{\text {out }}} E_{i j}^{\prime}-N_{\text {out }} \sum_{i}^{N_{\text {in }}} E_{i}^{\prime}-N_{\text {in }} \sum_{i}^{N_{\text {out }}} E_{i}^{\prime}
\end{gathered}
$$

in which $E_{i}^{\prime}$ and $E_{i j}^{\prime}$ are calculated in an electrostatically embedding field of fixed point charges

$$
\triangle E_{i j}^{\prime}=E_{i j}^{\prime}-E_{i}^{\prime}-E_{j}^{\prime}
$$

while, the intra-layer energy of the outer region, i.e. $E($ out $)$, is truncated at the three-body interaction level and treated with the ML models without electrostatically embedding

$$
E(\text { out })=\sum_{i<j<k}^{N_{\text {out }}} E_{i j k}-\left(N_{\text {out }}-3\right) \sum_{i<j}^{N_{\text {out }}} E_{i j}+\frac{\left(N_{\text {out }}-2\right)\left(N_{\text {out }}-3\right)}{2} \sum_{i}^{N_{\text {out }}} E_{i} \text {. }
$$

Because the ML models are very efficient, the inclusion of the three-body interaction merely adds a little computational cost. Compared with the ML-MLEBF method including all three-body interaction in $E($ in $), E($ in/out $)$, and 
$E($ out $)$ in Eqs. 6-9 in the main text, in the above simplified ML-MLEBF method, only $E$ (out) contains the threebody interaction in Eq. S23 while $E($ in $)$ and $E($ in $/$ out $)$ merely does the two-body interaction. When the number of monomers within the inner region is fixed, the numbers of monomers and dimers in $E($ in $)$ and $E$ (in/out) are increased in a nearly linear-scaling way. Further improvements on ML-MLEBF, e.g. only including the two-body interaction for the intra-layer energy of the outer region, is under progress.
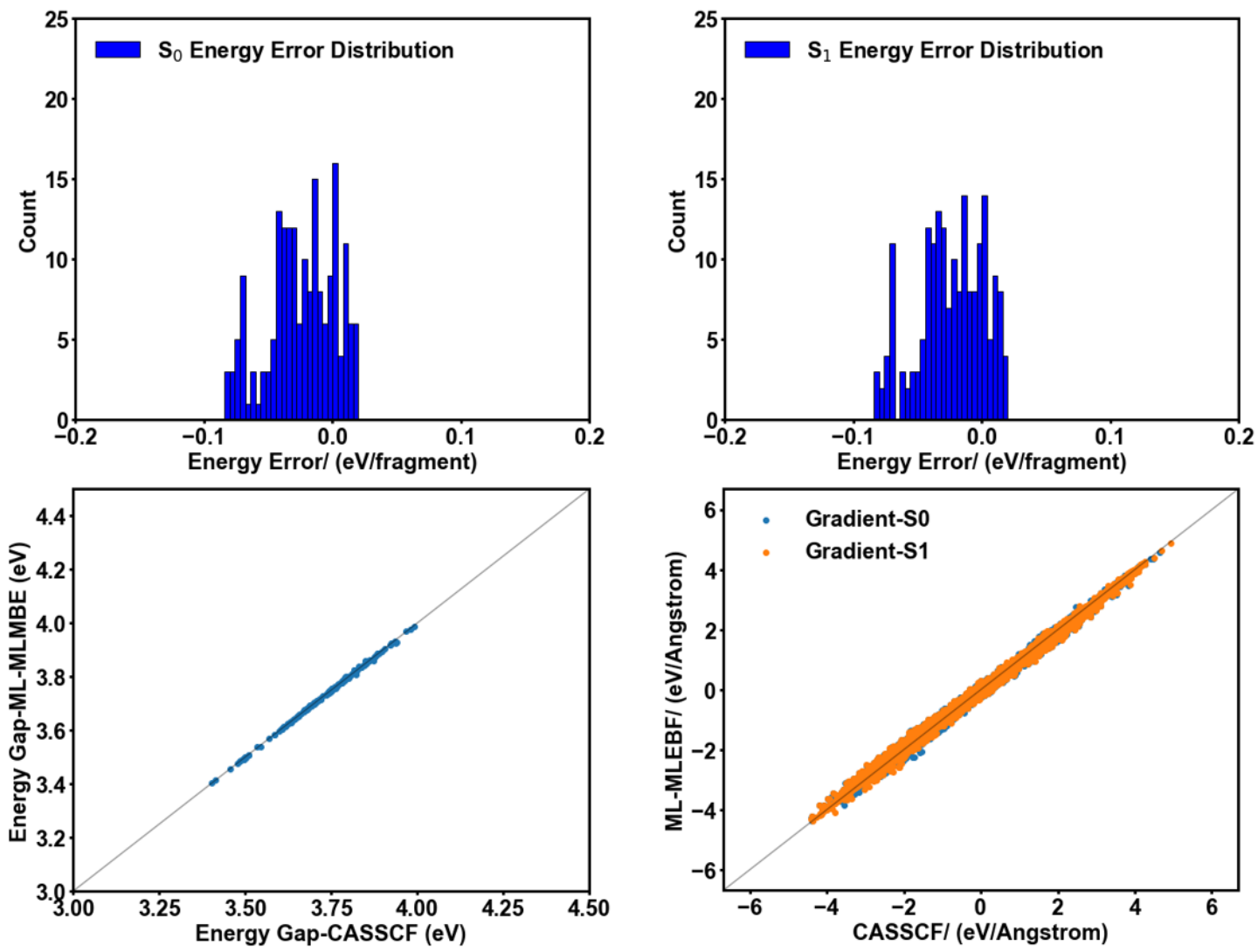

Figure S7: Comparison of both $S_{0}$ and $S_{1}$ energies (top-left and -right), energy gaps (bottom-left), and both $S_{0}$ and $S_{1}$ gradients (bottom-right) calculated with the simplified ML-MLEBF and full CASSCF methods. In this hybrid scheme, the intra-layer interaction of the inner region and the inter-layer interaction between the inner and outer regions are truncated at the two-body interaction with electrostatically embedding; whereas, the intra-layer interaction of the outer region is still truncated at the third-body interaction without electrostatically embedding because of $\mathrm{ML}$ calculations are extremely cheap.

Table S3: Computational Times (in second) of Single-Point Energies of a $\mathrm{CH}_{3} \mathrm{~N}=\mathrm{NCH}_{3}$ Chromophore Solvated with 10, 20, 30, or 40 Water Molecules using 1, 4, and 10 CPUs with both Simplified MLEBF and ML-MLEBF methods.

\begin{tabular}{|l|c|c|c|c|}
\hline & $10 \mathrm{H}_{2} \mathrm{O}$ & $20 \mathrm{H}_{2} \mathrm{O}$ & $30 \mathrm{H}_{2} \mathrm{O}$ & $40 \mathrm{H}_{2} \mathrm{O}$ \\
\hline \hline 1 CPU MLEBF & 2483 & 16251 & 57420 & 142712 \\
\hline 4 CPU MLEBF & 624 & 4163 & 14562 & 35874 \\
\hline 10 CPU MLEBF & 251 & 1675 & 5802 & 14302 \\
\hline 1 CPU ML-MLEBF & 871 & 2437 & 6067 & 13809 \\
\hline 4 CPU ML-MLEBF & 258 & 667 & 1576 & 3766 \\
\hline 10 CPU ML-MLEBF & 89 & 251 & 615 & 1407 \\
\hline
\end{tabular}


This simplification significantly reduces the computational cost but the results still are as accurate as those with the electrostatically embedding MLEBF method truncated at the two-body interaction level (EE2B-MLEBF in our previous work ${ }^{13}$ and Fig. S7). RMSDs of $S_{0}$ and $S_{1}$ energies [gradients] are $3.54 \times 10^{-2} \mathrm{eV} /$ fragment $\left[8.51 \times 10^{-2} \mathrm{eV} / \AA \AA A\right]$ and $3.52 \times 10^{-2} \mathrm{eV} /$ fragment $\left[8.48 \times 10^{-2} \mathrm{eV} / \mathrm{A}\right] . \mathrm{RMSD}$ of excitation energies is calculated to be $3.32 \times 10^{-3} \mathrm{eV}$. The speedup of this simplified ML-MLEBF method is significant. As discussed in the main text, it is highly parallel and efficient. Compared with MLEBF, it significantly reduces the computational cost (Fig. 5 in the main text and Table S3). Of course, ML-MLEBF and MLEBF method is also much more efficient than full CASSCF calculations, in particular for large systems (Fig. S8).Fig. S9 and Fig. S10 clearly show the results of energies and gradients of both simplified ML-MLEBF and EE2B-MLEBF methods. Both kinds of methods give more or less similar results.
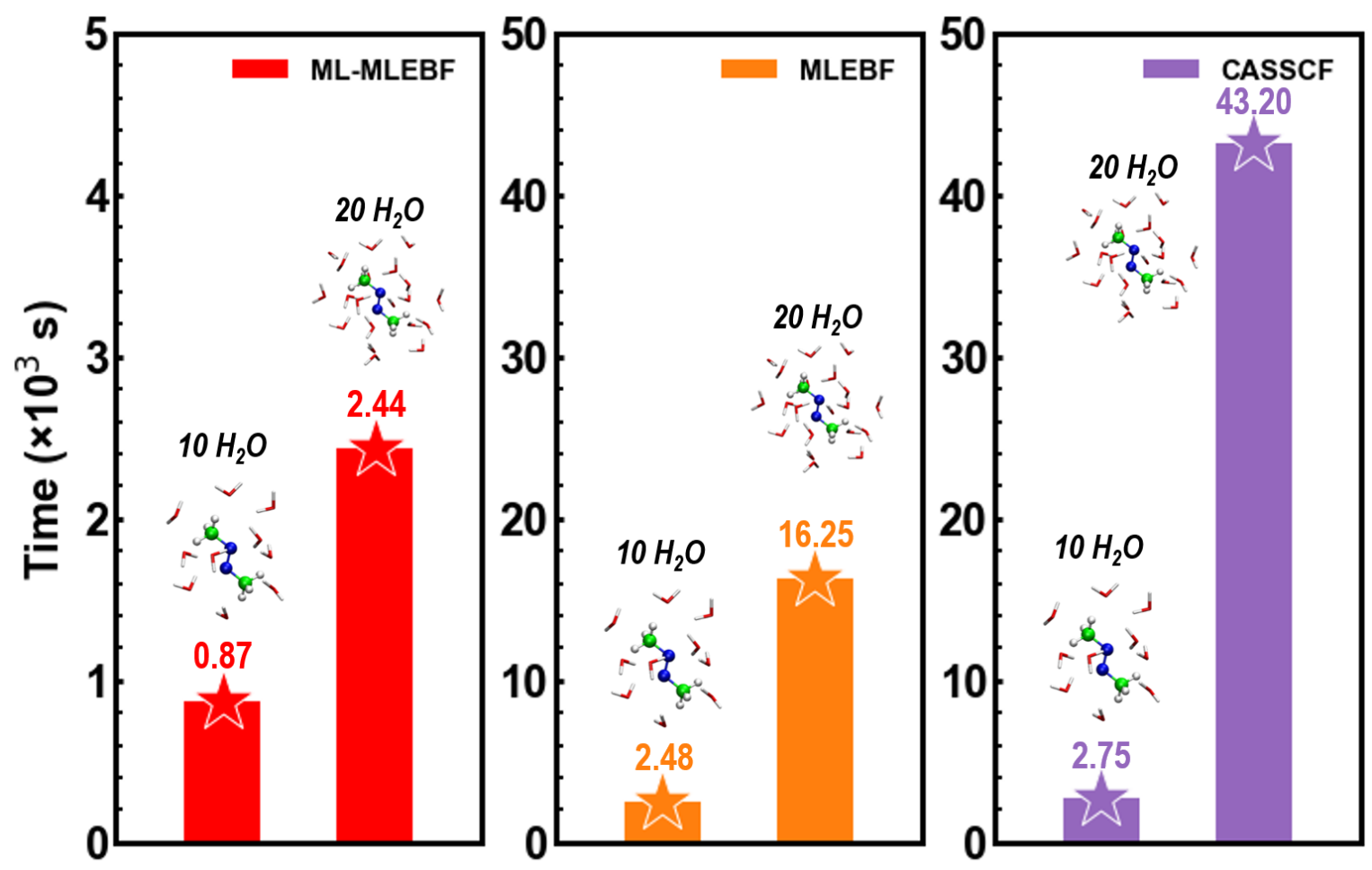

Figure S8: Computational times (in second) of single-point energies and gradients of $\mathrm{CH}_{3} \mathrm{~N}=\mathrm{NCH}_{3}$ chromophore solvated with 10 or 20 water molecules using 1 CPU with MLEBF, ML-MLEBF, and full CASSCF. It should be noted that $\mathrm{CH}_{3} \mathrm{~N}=\mathrm{NCH}_{3}$ chromophore with 30 or 40 water molecules has totally about 100 or 130 atoms for which state-averaged full CASSCF gradient calculations become difficult with general computer clusters.

\section{Comparison between MLEBF and QM/MM method}

The QM/MM results are less accurate compared with the full CASSCF ones with RMSDs of 0.101 and 0.104 $\mathrm{eV} /$ monomer for $\mathrm{S}_{0}$ and $\mathrm{S}_{1}$ [gradients: 1.074 and $1.076 \mathrm{eV} / \mathrm{A}$ ]. The $\mathrm{QM} / \mathrm{MM}$ calculated $\mathrm{S}_{1}-\mathrm{S}_{0}$ energy gaps are 

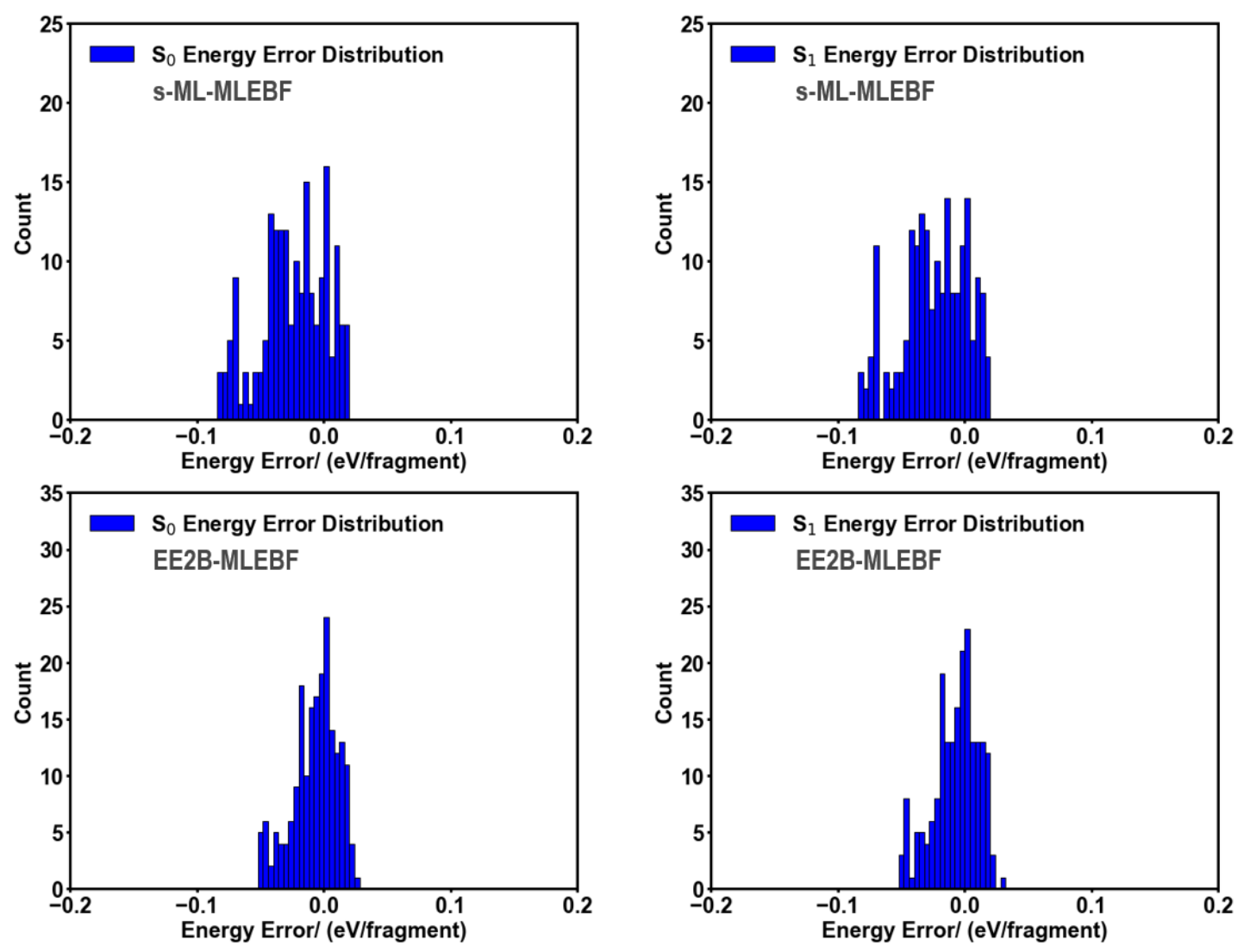

Figure S9: Error distributions of energies for simplified ML-MLEBF (top) and EE2B-MLEBF methods (bottom). 

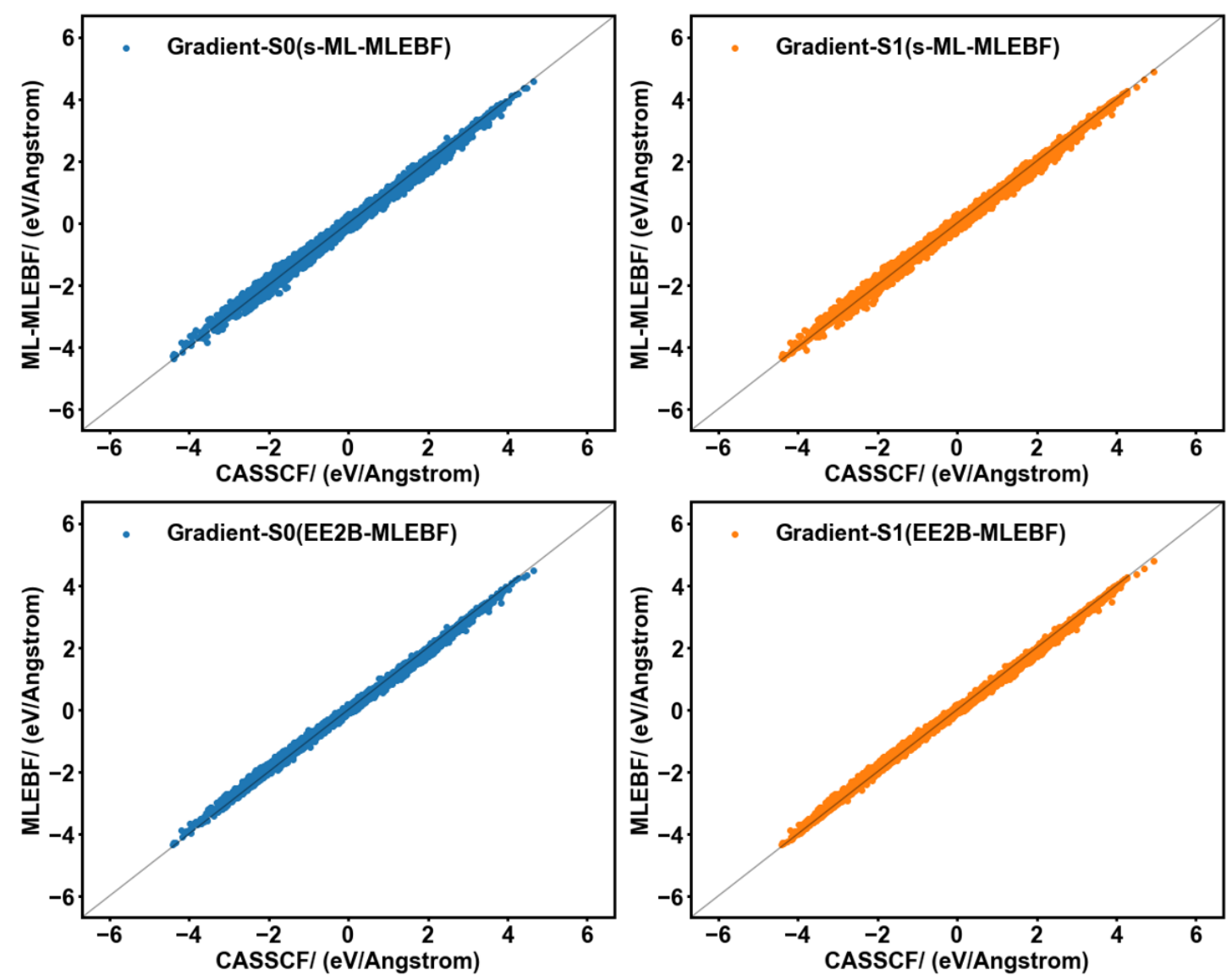

Figure S10: Comparison of gradients calculated using simplified ML-MLEBF and CASSCF methods (top) and comparison of gradients calculated using EE2B-MLEBF and CASSCF methods (bottom). 

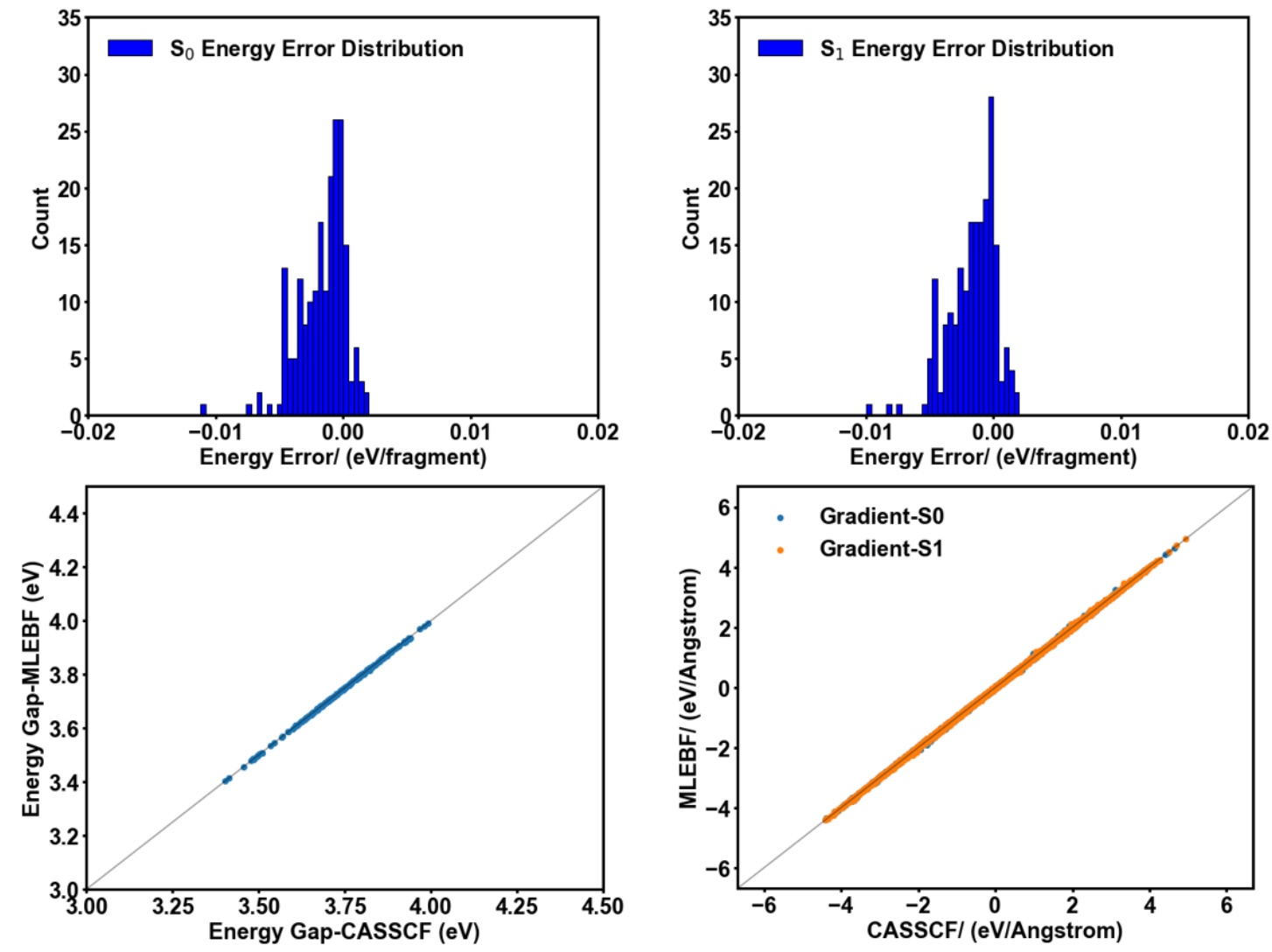

Figure S11: Comparison of both $S_{0}$ and $S_{1}$ energies (top-left and -right), energy gaps (bottom-left), and both $\mathrm{S}_{0}$ and $\mathrm{S}_{1}$ gradients (bottom-right) calculated with MLEBF with despersion correction and full CASSCF methods. In this scheme, the intra-layer interaction of the inner region and the inter-layer interaction between the inner and outer regions are truncated at the three-body interaction level without electrostatically embedding; whereas, the intra-layer interaction of the outer region is still truncated at the third-body interaction level without electrostatically embedding at the B3LYP-D3/6-31+G** level. 

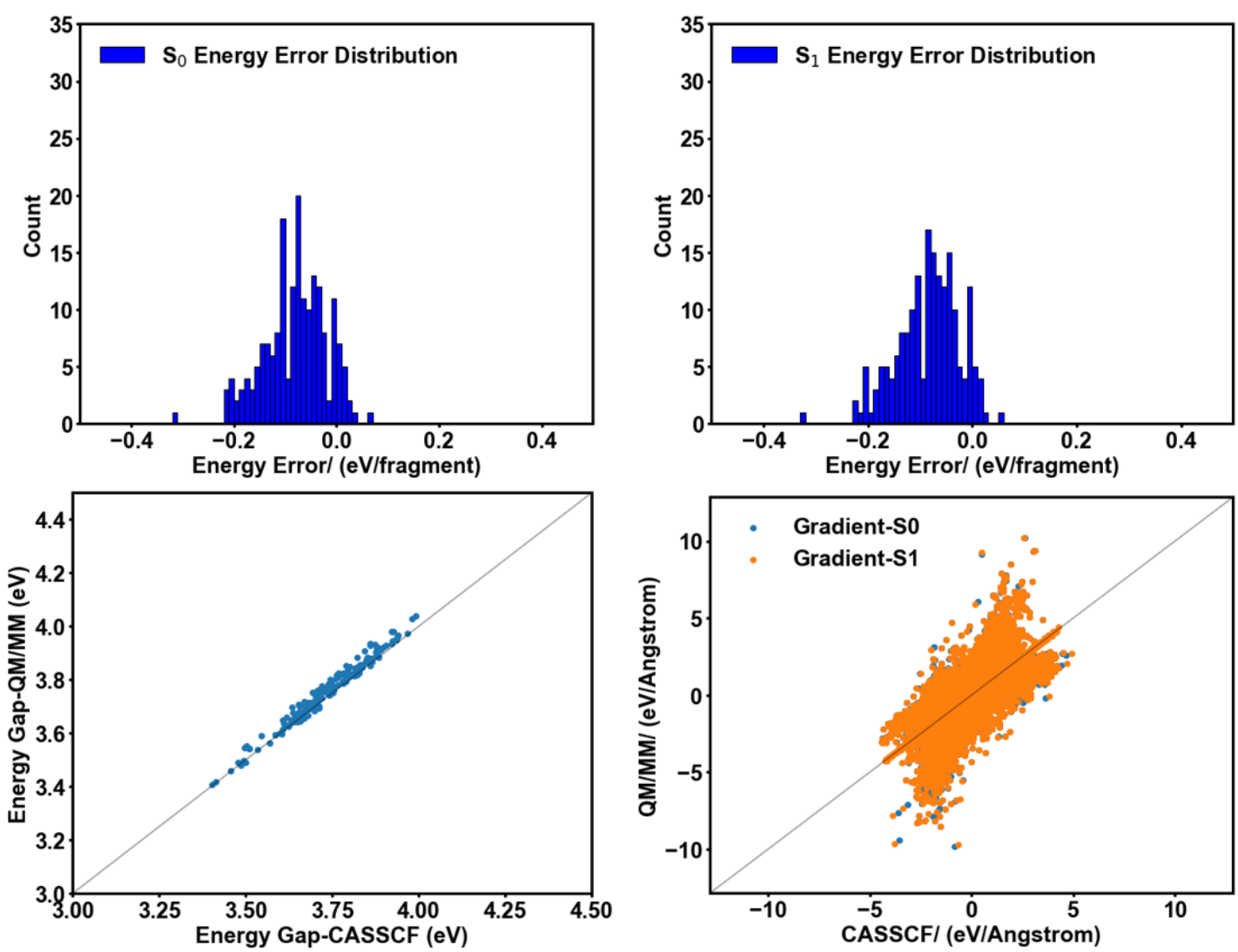

Figure S12: Comparison of both $\mathrm{S}_{0}$ and $\mathrm{S}_{1}$ energies (top-left and -right), energy gaps (bottom-left), and both $\mathrm{S}_{0}$ and $\mathrm{S}_{1}$ gradients (bottom-right) calculated using QM/MM and CASSCF for $\mathrm{CH}_{3} \mathrm{~N}=\mathrm{NCH}_{3}-\left(\mathrm{H}_{2} \mathrm{O}\right)_{5}$ system.

accurate enough with RMSD of $2.91 \times 10^{-2} \mathrm{eV}$ (see Fig. S12). 


\section{References}

(1) Perdew, J. P. Density-Functional Approximation for the Correlation Energy of the Inhomogeneous Electron Gas. Phys. Rev. B 1986, 33, 8822-8824.

(2) Becke, A. D. Density-Functional Exchange-Energy Approximation with Correct Asymptotic Behavior. Phys. Rev. A 1988, 38, 3098-3100.

(3) Weigend, F.; Ahlrichs, R. Balanced Basis Sets of Split Valence, Triple Zeta Valence and Quadruple Zeta Valence Quality for H to Rn: Design and Assessment of Accuracy. Phys. Chem. Chem. Phys. 2005, 7, 3297-3305.

(4) Nosé, S. A Unified Formulation of the Constant Temperature Molecular Dynamics Methods. J. Chem. Phys. 1984, 81, 511-519.

(5) Hoover, W. G. Canonical Dynamics: Equilibrium Phase-Space Distributions. Phys. Rev. A 1985, 31, 16951697.

(6) ChemShell3.5, a Computational Chemistry Shell, see www.chemshell.org.

(7) Sherwood, P.; de Vries, A. H.; Guest, M. F.; Schreckenbach, G.; Catlow, C. A.; French, S. A.; Sokol, A. A.; Bromley, S. T.; Thiel, W.; Turner, A. J.; et al., QUASI: A General Purpose Implementation of the QM/MM Approach and its Application to Problems in Catalysis. J. Mol. Struct.: THEOCHEM 2003, 632, 1-28.

(8) Metz, S.; Kastner, J.; Sokol, A. A.; Keal, T. W.; Sherwood, P. Chemshell-A Modular Software Package for QM/MM Simulations. Wiley Interdiscip. Rev.: Comput. Mol. Sci. 2014, 4, 101-110.

(9) TURBOMOLE V6.6, 2014, a development of University of Karlsruhe and Forschungszentrum Karlsruhe GmbH, 1989-2007,TURBOMOLE GmbH, since 2007; available from http://www . turbomole. com.

(10) Ahlrichs, R.; Bär, M.; Häser, M.; Horn, H.; Kölmel, C. Electronic Structure Calculations on Workstation Computers: The Program System Turbomole. Chem. Phys. Lett. 1989, 162, 165-169.

(11) Furche, F.; Ahlrichs, R.; Hättig, C.; Klopper, W.; Sierka, M.; Weigend, F. Turbomole. Wiley Interdiscip. Rev.: Comput. Mol. Sci. 2014, 4, 91-100.

(12) Ruckenbauer, M.; Barbatti, M.; Sellner, B.; Muller, T.; Lischka, H. Azomethane: Nonadiabatic Photodynamical Simulations in Solution. J. Phys. Chem. A 2010, 114, 12585-12590.

(13) Chen, W. K.; Fang, W. H.; Cui, G. L. Multi-Layer Energy-Based Fragment Method for Excited States and Nonadiabatic Dynamics. Phys. Chem. Chem. Phys. 2019, 21, 22695-22699. 
(14) Karlström, G.; Lindh, R.; Malmqvist, P.; Roos, B. O.; Ryde, U.; Veryazov, V.; Widmark, P.-O.; Cossi, M.; Schimmelpfennig, B.; Neogrady, P.; et al., MOLCAS: A Program Package for Computational Chemistry. Comput. Mater. Sci. 2003, 28, 222-239.

(15) Aquilante, F.; Autschbach, J.; Carlson, R. K.; Chibotaru, L. F.; Delcey, M. G.; De Vico, L.; Fdez. Galván, I.; Ferré, N.; Frutos, L. M.; Gagliardi, L.; et al., Molcas 8: New Capabilities for Multiconfigurational Quantum Chemical Calculations across the Periodic Table: Molcas 8. J. Comput. Chem. 2016, 37, 506-541.

(16) Behler, J.; Parrinello, M. Generalized Neural-Network Representation of High-Dimensional PotentialEnergy Surfaces. Phys. Rev. Lett. 2007, 98, 146401.

(17) Rupp, M.; Tkatchenko, A.; Müller, K.-R.; von Lilienfeld, O. A. Fast and Accurate Modeling of Molecular Atomization Energies with Machine Learning. Phys. Rev. Lett. 2012, 108, 058301.

(18) Jiang, B.; Guo, H. Permutation Invariant Polynomial Neural Network Approach to Fitting Potential Energy Surfaces. J. Chem. Phys. 2013, 139, 054112.

(19) Chen, J.; Xu, X.; Xu, X.; Zhang, D. H. A Global Potential Energy Surface for the $\mathrm{H}_{2}+\mathrm{OH} \leftrightarrow \mathrm{H}_{2} \mathrm{O}+\mathrm{H}$ Reaction Using Neural Networks. J. Chem. Phys. 2013, 138, 154301.

(20) Chen, J.; Xu, X.; Xu, X.; Zhang, D. H. Communication: An Accurate Global Potential Energy Surface for the $\mathrm{OH}+\mathrm{CO} \leftrightarrow \mathrm{H}+\mathrm{CO}_{2}$ Reaction Using Neural Networks. J. Chem. Phys. 2013, 138, 221104.

(21) Shao, K. J.; Chen, J.; Zhao, Z. Q.; Zhang, D. H. Communication: Fitting Potential Energy Surfaces with Fundamental Invariant Neural Network. J. Chem. Phys. 2016, 145, 071101.

(22) Behler, J. First Principles Neural Network Potentials for Reactive Simulations of Large Molecular and Condensed Systems. Angew. Chem., Int. Ed. 2017, 56, 12828-12840.

(23) Liu, Q.; Wang, J. C.; Du, P. L.; Hu, L. H.; Zheng, X.; Chen, G. H. Improving the Performance of LongRange-Corrected Exchange-Correlation Functional with an Embedded Neural Network. J. Phys. Chem. A 2017, 121, 7273-7281.

(24) Guan, Y. F.; Fu, B. N.; Zhang, D. H. Construction of Diabatic Energy Surfaces for LiFH with Artificial Neural Networks. J. Chem. Phys. 2017, 147, 224307.

(25) Zhang, L.; Han, J.; Wang, H.; Car, R.; E, W. Deep Potential Molecular Dynamics: A Scalable Model with the Accuracy of Quantum Mechanics. Phys. Rev. Lett. 2018, 120, 143001.

(26) Hu, D.; Xie, Y.; Li, X.; Li, L.; Lan, Z. Inclusion of Machine Learning Kernel Ridge Regression Potential Energy Surfaces in On-the-Fly Nonadiabatic Molecular Dynamics Simulation. J. Phys. Chem. Lett. 2018, $9,2725-2732$. 
(27) Dral, P. O.; Barbatti, M.; Thiel, W. Nonadiabatic Excited-State Dynamics with Machine Learning. J. Phys. Chem. Lett. 2018, 9, 5660-5663.

(28) Chen, W. K.; Liu, X. Y.; Fang, W. H.; Dral, P. O.; Cui, G. L. Deep Learning for Nonadiabatic Excited-State Dynamics. J. Phys. Chem. Lett. 2018, 9, 6702-6708.

(29) Wang, H.; Yang, W. Force Field for Water Based on Neural Network. J. Phys. Chem. Lett. 2018, 9, 32323240.

(30) Fu, B. N.; Zhang, D. H. Ab Initio Potential Energy Surfaces and Quantum Dynamics for Polyatomic Bimolecular Reactions. J Chem. Theory Comput. 2018, 14, 2289-2303.

(31) Wang, H.; Yang, W. T. Toward Building Protein Force Fields by Residue-Based Systematic Molecular Fragmentation and Neural Network. J. Chem. Theory Comput. 2018, 15, 1409-1417.

(32) Zhang, Y. L.; Hu, C.; Jiang, B. Embedded Atom Neural Network Potentials: Efficient and Accurate Machine Learning with a Physically Inspired Representation. J. Phys. Chem. Lett. 2019, 10, 4962-4967.

(33) Xu, M. Y.; Zhu, T.; Zhang, J. Z. H. Molecular Dynamics Simulation of Zinc Ion in Water with an ab Initio Based Neural Network Potential. J. Phys. Chem. A 2019, 123, 6587-6595.

(34) Berendsen, H. J. C.; Postma, J. P. M.; Van Gunsteren, W. F.; Hermans, J. Intermolecular Forces; Reidel, Dordrecht Jerusalem, Israel; 1981.

(35) Paesani, F.; Zhang, W.; Case, D. A.; Cheatham, T. E.; Voth, G. A. An Accurate and Simple Quantum Model for Liquid Water. J. Chem. Phys. 2006, 125, 184507.

(36) Lee, C.; Yang, W.; Parr, R. G. Development of the Colle-Salvetti Correlation-Energy Formula into a Functional of the Electron Density. Phys. Rev. B 1988, 37, 785.

(37) Vosko, S. H.; Wilk, L.; Nusair, M. Accurate Spin-Dependent Electron Liquid Correlation Energies for Local Spin Density Calculations: A Critical Analysis. Can. J. Phys. 1980, 58, 1200-1211.

(38) Ditchfield, R.; Hehre, W. J.; Pople, J. A. Self-Consistent Molecular-Orbital Methods. IX. An Extended Gaussian-Type Basis for Molecular-Orbital Studies of Organic Molecules. J. Chem. Phys. 1971, 54, 724728.

(39) Hehre, W. J.; Ditchfield, R.; Pople, J. A. Self-Consistent Molecular Orbital Methods. XII. Further Extensions of Gaussian-Type Basis Sets for Use in Molecular Orbital Studies of Organic Molecules. J. Chem. Phys. $1972,56,2257-2261$. 
(40) Hariharan, P. C.; Pople, J. A. The Influence of Polarization Functions on Molecular Orbital Hydrogenation Energies. Theor. Chim. Acta 1973, 28, 213-222.

(41) Clark, T.; Chandrasekhar, J.; Spitznagel, G. W.; Schleyer, P. V. R. Efficient Diffuse Function-Augmented Basis Sets for Anion Calculations. III. The 3-21+G Basis Set for First-Row Elements, Li-F. J. Comput. Chem. 1983, 4, 294-301.

(42) Case, D. A.; Cerutti, D. S.; Cheatham III, T. E.; Darden, T. A.; Duke, R. E.; Giese, T. J.; Gohlke, H.; Goetz, A.; Greene, D.; Homeyer, N.; et al., AMBER 2017; University of California: San Francisco, CA, USA, 2017.

(43) Frisch, M.; Trucks, G.; Schlegel, H.; Scuseria, G.; Robb, M.; Cheeseman, J. R.; Scalmani, G.; Barone, V.; Petersson, G.; Nakatsuji, H.; et al., Gaussian 16, Revision A. 03. Gaussian, Inc., Wallingford CT, 2016.

(44) Wang, H.; Zhang, L.; Han, J.; E, W. DeePMD-kit: A Deep Learning Package For Many-body Potential Energy Representation and Molecular Dynamics. Comput. Phys. Commun. 2018, 228, 178-184.

(45) Zhu, C.; Nakamura, H. Theory of Nonadiabatic Transition for General Two-State Curve Crossing Problems. I. Nonadiabatic Tunneling Case. J. Chem. Phys. 1994, 101, 10630-10647.

(46) Zhu, C.; Nakamura, H. Theory of Nonadiabatic Transition for General Two-State Curve Crossing Problems. II. Landau-Zener Case. J. Chem. Phys. 1995, 102, 7448-7461.

(47) Zhu, C.; Nobusada, K.; Nakamura, H. New Implementation of the Trajectory Surface Hopping Method with Use of the Zhu-Nakamura Theory. J. Chem. Phys. 2001, 115, 3031-3044.

(48) Yu, L.; Xu, C.; Lei, Y.; Zhu, C.; Wen, Z. Trajectory-Based Nonadiabatic Molecular Dynamics without Calculating Nonadiabatic Coupling in the Avoided Crossing Case: Trans $\leftrightarrow$ Cis Photoisomerization in Azobenzene. Phys. Chem. Chem. Phys. 2014, 16, 25883-25895.

(49) Yu, L.; Xu, C.; Zhu, C. Probing the $\pi \rightarrow \pi^{*}$ Photoisomerization Mechanism of Cis-Azobenzene by MultiState ab initio On-the-Fly Trajectory Dynamics Simulation. Phys. Chem. Chem. Phys. 2015, 17, 1764617660.

(50) Wigner, E. On the Quantum Correction for Thermodynamic Equilibrium. Phys. Rev. 1932, 40, 749-759.

(51) Barbatti, M.; Granucci, G.; Persico, M.; Ruckenbauer, M.; Vazdar, M.; Eckert-Maksić, M.; Lischka, H. The On-the-Fly Surface-Hopping Program System Newton-X: Application to ab initio Simulation of the Nonadiabatic Photodynamics of Benchmark Systems. J. Photochem. Photobiol. A 2007, 190, $228-240$.

(52) Barbatti, M.; Ruckenbauer, M.; Plasser, F.; Pittner, J.; Granucci, G.; Persico, M.; Lischka, H. Newton-X: A Surface-Hopping Program for Nonadiabatic Molecular Dynamics. Wiley Interdiscip. Rev.: Comput. Mol. Sci. 2014, 4, 26-33. 
(53) Cui, G. L.; Thiel, W. Generalized Trajectory Surface-Hopping Method for Internal Conversion and Intersystem Crossing. J. Chem. Phys. 2014, 141, 124101. 